\title{
Measurement of $\Delta S$ in the Nucleon from Semi-inclusive DIS on Deuterium
}

\author{
H. E. Jackson \\ Argonne National Laboratory, Argonne, IL 60439 \\ (on behalf of the HERMES collaboration)
}

\begin{abstract}
The helicity density of the strange quark sea in the nucleon has been extracted from measurements of semi-inclusive polarized deep-inelastic scattering on the deuteron. In the region of measurement the helicity density is zero within experimental error, and in a leading-order analysis the integral of the density over the region $x>0.02$ is determined to be $0.006 \pm 0.029$ (stat.) \pm 0.007 (sys.). The first moment of the axial charge over the same region is substantially less than that inferred from hyperon-semi-leptonic decays.
\end{abstract}

Keywords: strange quarks, nucleon spin, deep-inelastic scattering PACS: $13.60 .-\mathrm{r}, 13.88 .+\mathrm{e}, 14.20 . \mathrm{Dh}, 14.65 . \mathrm{-q}$

\section{INTRODUCTION}

The polarization of the strange quark sea is of great interest as a probe of the spin properties of the quark sea in the nucleon. Because strange quarks carry no isospin, the total strange quark helicity density $\Delta S(x) \equiv \Delta s(x)+\Delta \bar{s}(x)$, where $x$ is the Bjorken scaling variable, can be extracted from polarization measurements in scattering on the isoscalar deuteron alone. The helicity density $\Delta Q(x) \equiv \Delta u(x)+\Delta \bar{u}(x)+\Delta d(x)+\Delta \bar{d}(x)$ of the nonstrange sea can be estimated from measurement of the inclusive spin asymmetries. Using the spin asymmetries measured for the charged kaons as the second experimental data set, it is possible to extract $\Delta S(x)$. By measuring the charged kaon multiplicities with the same data, the fragmentation functions relevant to the extraction process can be obtained without resort to other experiments. Aside from that of isospin symmetry between the proton and neutron, the only assumption required in the analysis is chargeconjugation invariance in the fragmentation process. A precise "isoscalar" extraction of $\Delta \mathrm{S}(\mathrm{x})$ using semi-inclusive DIS on the deuteron has been carried out at HERMES.

\section{EXPERIMENT}

The asymmetries were measured by the HERMES experiment using a longitudinally polarized deuteron gas target internal to the $\mathrm{E}=27.5 \mathrm{GeV}$ HERA positron storage ring at DESY. The self-induced beam polarization is measured continuously with Compton backscattering of circularly polarized laser beams $[1,2]$. The open-ended target cell is fed by an atomic-beam source based on Stern-Gerlach separation [3] with hyperfine transitions. The nuclear polarization of the atoms is randomly chosen at $90 \mathrm{~s}$ time intervals, while both this polarization and the atomic fraction inside the target cell are continu-

\footnotetext{
CP915, Proceedings of the $17^{\text {th }}$ International Spin Physics Symposium, edited by K. Imai, T. Murakami, N. Saito, and K. Tanida
} O 2007 American Institute of Physics 978-0-7354-0423-6/07/\$23.00 
ously measured $[4,5]$. Scattered beam leptons and coincident hadrons are detected by the HERMES spectrometer [6]. Leptons are identified with an efficiency exceeding $98 \%$ and a hadron contamination of less than $1 \%$ using an electromagnetic calorimeter, a transition-radiation detector, a preshower scintillation counter and a Čerenkov detector. Charged kaons are identified using a dual-radiator ring-imaging Črenkov detector [7]. Events were selected subject to the kinematic requirements $Q^{2}>1 \mathrm{GeV}^{2}, W^{2}>10 \mathrm{GeV}^{2}$ and $y<0.85$, where $Q^{2}$ is the negative squared 4-momentum transfer, $W$ is the invariant mass of the photon-nucleon system, and $y=v / E$. Coincident hadrons were accepted if $0.2<z<0.8$ and $x_{F} \approx 2 p_{L} / W>0.1$, where $p_{L}$ is the longitudinal momentum of the hadron with respect to the virtual photon direction in the photon-nucleon center of mass frame.

\section{ANALYSIS}

The fragmentation functions integrated over the range in $z=E_{K} / v$ required for the extraction of the helicity densities have been obtained directly from the same polarized data set for the deuteron that has been used to extract the double spin asymmetries by averaging the data for the two spin states. In leading order in $\alpha_{s}$ and assuming only charge conjugation invariance for charged kaon production in semi-inclusive DIS on the deuteron

$$
\frac{d N^{K}(x) / d x}{d N^{d i s}(x) / d x}=\frac{Q(x) \int \mathscr{D}_{Q}^{K}(z) d z+S(x) \int \mathscr{D}_{S}^{K}(z) d z}{5 Q(x)+2 S(x)} .
$$

The strange and non-strange fragmentation integrals can be extracted by fitting the $x$ dependence of this ratio using Eq. 1 together with parton distributions taken from the CTEQ6 compilation [8]. A fit to the $\mathrm{K}^{ \pm}$multiplicity extracted using Eq. 1 with the limits of integration $0.2<\mathrm{z}<0.8$ is presented in Fig. 1. The fit gives $\int_{0.2}^{0.8} \mathscr{D}_{Q}^{K}(z) d z=$ $0.41 \pm 0.02($ stat. $) \pm 0.03($ sys. $)$ and $\int_{0.2}^{0.8} \mathscr{D}_{S}^{K}(z) d z=1.41 \pm 0.29$ (stat. $) \pm 0.15$ (sys.).

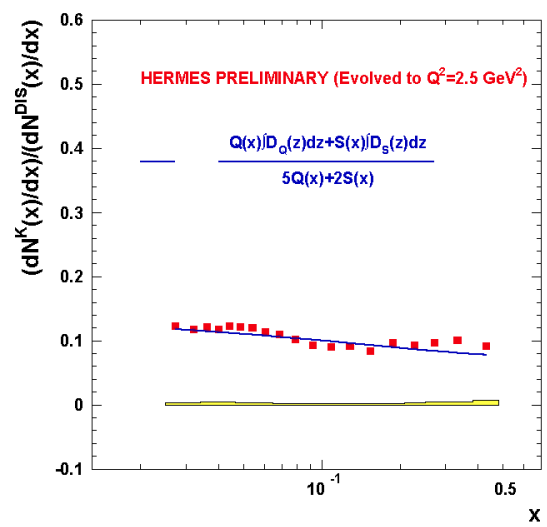

FIGURE 1. Multiplicity in $4 \pi$, corrected for acceptance and smearing effects, of charged kaons in semiinclusive DIS on a deuterium target as a function of Bjorken $x$. The statistical error bars are not visible, and the bands at the bottom represent the systematic uncertainties. 

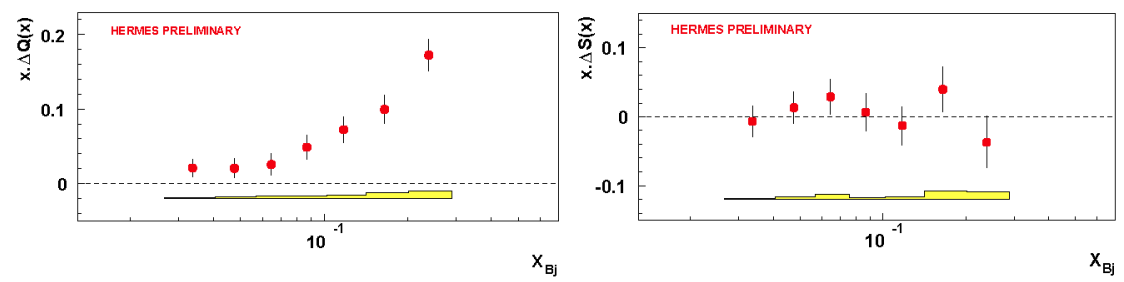

FIGURE 2. Strange and non-strange quark helicity distributions at $\left\langle Q^{2}\right\rangle=2.5 \mathrm{GeV}^{2}$ as a function of Bjorken $x$. The error bars are statistical, and the bands at the bottom represent the systematic uncertainties.

The inclusive and semi-inclusive charged-kaon double-spin asymmetries for a deuteron target in leading order when integrated over the range $0.2<z<0.8$ are

$$
A_{1, d}^{K}(x)=\frac{\Delta Q(x) \int \mathscr{D}_{Q}^{K}(z) d z+\Delta S(x) \int \mathscr{D}_{S}^{K}(z) d z}{Q(x) \int \mathscr{D}_{Q}^{K}(z) d z+S(x) \int \mathscr{D}_{S}^{K}(z) d z}, A_{1, d}(x)=\frac{5 \Delta Q(x)+2 \Delta S(x)}{5 Q(x)+2 S(x)} .
$$

The helicity distributions $\Delta Q(x)$ and $\Delta S(x)$ can be extracted directly from the measured values of $A_{1, d}(x)$ and $A_{1, d}^{K^{ \pm}}(x)$ using the fragmentation integrals extracted from the charged kaon multiplicities and the parton distributions $\mathrm{Q}(\mathrm{x})$ and $\mathrm{S}(\mathrm{x})$ taken from the latest CTEQ6L compilation [8].

\section{RESULTS}

The strange and non-strange helicity distributions weighted by $x$ obtained in the HERMES leading-order analysis are presented in Fig. 2. The non-strange helicity distribution is in excellent agreement with that derived from the published five-component flavor decomposition [9] of the proton helicity. While of much improved precision and free of the systematic uncertainties in the fragmentation functions, the strange helicity distribution also agrees well with the results reported therein, and is consistent with zero over the measured range.

The integrals of the measured distributions in the measured range of Bjorken $x$ are given in Table 1. The integral over the measured region of $\Delta \mathrm{S}(\mathrm{x})$ is consistent with zero. Because of the very small density of strange quarks above $x=0.3$ the contribution of any non-zero helicity density in this region is negligible compared to the systematic error in the measurement. Consequently, the value for $\Delta \mathrm{S}$ can be safely taken as the integral over the Bjorken $\mathrm{x}$ range $0.02-1.0$. This conclusion contrasts those of the early

TABLE 1. Integrals of various helicity distributions in the $x$ Bjorken range 0.02-0.6

\begin{tabular}{lr}
\hline & Integral in measured range \\
\hline$\Delta Q$ & $0.286 \pm 0.026$ (stat.) \pm 0.011 (sys.) \\
$\Delta S$ & $0.006 \pm 0.029$ (stat.) \pm 0.007 (sys.) \\
$\Delta q_{8}$ & $0.274 \pm 0.039$ (stat.) \pm 0.018 (sys.) \\
\hline
\end{tabular}


inclusive measurements $[10,11]$ which reported a substantial negative polarization for the strange quarks. It suggests that the violation of the Ellis-Jaffe sum rule observed in these inclusive measurements is not the result of a significant negative polarization of the strange sea.

The results for the integrals can also be used to study directly the assumption made in the analysis of inclusive DIS data, namely SU(3) symmetry in hyperon decays. The octet combination $\Delta q_{8}(x)=\Delta Q(x)-2 \Delta S(x)$ can be extracted from the integrals of $\mathrm{Q}(\mathrm{x})$ and $\mathrm{S}(\mathrm{x})$. The isoscalar result is included in Table 1 . The result is to be compared with the value of the first moment extracted under the assumption of SU(3) symmetry from the hyperon decay constants [12] $a_{8}=(3 F-D) C_{Q C D}=0.49$. The difference from the isoscalar result of 0.274 must be found in the low $\mathrm{x}$ region $\mathrm{x}<0.02$ if $\mathrm{SU}(3)$ symmetry is to be required.

An estimate of the strange quark helicity distribution at high $\mathrm{Q}^{2}$ follows from the relationship [13]

$$
\Delta S=G_{A}^{s}\left(Q^{2}=0\right)=\int_{0}^{1} \Delta S\left(x, Q^{2}=\infty\right) d x .
$$

In a combined analysis of parity violation measurements in elastic ep scattering and of elastic $v$ p scattering [13] $\mathrm{G}_{A}^{s}\left(\mathrm{Q}^{2}\right)$ has been extracted over the range $0.4 \mathrm{GeV}^{2}<\mathrm{Q}^{2}<1.0$ $\mathrm{GeV}^{2}$. While the data above $0.5 \mathrm{GeV}^{2}$ are consistent with zero the lowest measurement at $\mathrm{Q}^{2}=0.5 \mathrm{GeV}^{2}$ suggests that at very low momentum transfers the the axial form factor may become increasingly negative as $\mathrm{Q}^{2} \rightarrow 0$. The semi-inclusive DIS result reported here appears to contradict that conclusion. The discrepancy could be resolved by the onset of large values of $\Delta S\left(x, Q^{2}\right)$ at very low values of $x_{b j}, x \approx 10^{-2}-10^{-3}$.

\section{ACKNOWLEDGMENTS}

We gratefully acknowledge the DESY management for its support, the staff at DESY and the collaborating institutions for the significant effort, and our national funding agencies for their financial support.

\section{REFERENCES}

1. D. P. Barber, et al., Nucl. Inst. \& Meth. A 338, 166 (1994).

2. M. Beckmann, et al., Nucl. Inst. \& Meth. A 479, 334 (2002).

3. F. Stock, et al., Nucl. Inst. \& Meth. A 343, 334 (1994).

4. C. Baumgarten, et al., Nucl. Inst. \& Meth. A 482, 606 (2002).

5. C. Baumgarten, et al., Nucl. Inst. \& Meth. A 496, 263-276 (2003).

6. K. Ackerstaff, et al., Nucl. Inst. \& Meth. A 417, 230 (1998).

7. N. Akopov, et al., Nucl. Inst. \& Meth. A 479, 511 (2002).

8. J. Pumplin, et al., J. High Energy Phys. 7, 12 (2002).

9. A. Airapetian, et al., Phys. Rev. D 71, 012003 (2005).

10. J. Ashman, et al., Phys. Lett. B206, 364 (1988).

11. D. Adams, et al., Phys. Rev. D 56, 5330 (1997).

12. P. Ratcliffe, Czech J. Phys. 54, B11 (2004).

13. S. Pate, et al., in these proceedings (2006). 